\title{
SHADOWING, EXPANSIVENESS AND HYPERBOLIC HOMEOMORPHISMS
}

\author{
JERZY OMBACH \\ (Received 21 March 1994; revised 15 August 1994) \\ Communicated by P. E. Kloeden
}

\begin{abstract}
The purpose of this paper is to complete results concerning the class $\mathscr{H}$ of expansive homeomorphisms having the pseudo orbits tracing property on a compact metric space. We show that hyperbolic homeomorphisms introduced by Mañé in [8] are exactly those in the class $\mathscr{H}$; then by the result of $[12,20]$ they form a class equal to the Smale space introduced by Ruelle in [18]. Next, assuming that the phase space is a smooth manifold, we show that a diffeomorphism is Anosov if and only if it is in the class $\mathscr{H}$ and is a lower semi-continuity point of the map which assigns to any diffeomorphism the supremum of its expansive constants (possibly zero). Then we discuss the behavior of the dynamical systems generated by homeomorphisms from $\mathscr{H}$ near their basic sets.
\end{abstract}

1991 Mathematics subject classification (Amer. Math. Soc.): primary 58F15; secondary 54H20.

\section{Introduction}

Since Walters' paper [19] in 1978 many attempts have been made to express the concept of hyperbolicity in topological terms. Notions of shadowing, expansiveness, coordinates, Smale space and others have proved to be very useful in attaining this aim. A lot of results known for Anosov diffeomorphisms, Axiom A diffeomorphisms and, more generally, for diffeomorphisms on hyperbolic sets, as well as results known for the subshifts of finite type, have been extended to homeomorphisms defined on compact metric spaces and satisfying some of the above properties. We refer to the Aoki survey [2] the present author's paper [14], and Mañé's book [8, Section IV.9], for more information and complete references lists. In particular, in paper [12] the present author showed that many classes of homeomorphisms considered as topological counterparts of hyperbolicity were actually the same. So we can define

(C) 1996 Australian Mathematical Society 0263-6115/96 \$A2.00+0.00 
such a class requiring, for example, that any of its elements have the shadowing property and be expansive. Denote this class by $\mathscr{H}$.

The purpose of this paper is to complete results concerning the class $\mathscr{H}$, obtained by the author in $[12,13,14]$ and by others, see below. First we show that hyperbolic homeomorphisms introduced by Mañé in his book referred to above are exactly those in the class $\mathscr{H}$ (Theorem 3 ), then by the result of $[12,20]$ they form a class equal to the Smale space introduced by Ruelle in [18]. Next, assuming that the phase space is a smooth manifold, we compare the class $\mathscr{H}$ with the class of Anosov diffeomorphisms. In particular, a diffeomorphism is Anosov if and only if it is in the class $\mathscr{H}$ and is a lower semi-continuity point of the map which assigns to any diffeomorphism the supremum of its expansive constants (possibly zero); see below for the precise definition and the statement in Theorem 4. Moreover, the last condition is the essential one. Then we will discuss the behavior of the dynamical systems generated by homeomorphisms from $\mathscr{H}$ near their basic sets. Generally speaking, we will show that basic sets may admit exactly three kinds of behavior corresponding to these (and therefore named so) known as sink, source and saddle, while one considers a hyperbolic fixed point of a diffeomorphism (Sections 4 and 5). Finally, under assumptions of connectedness of the space, and taking advantage of the result from [17], we obtain extra interesting information on the nature of basic sets, Theorem 24. The results of Sections 4 and 5 have their partial counterparts in the continuous time case (for flows), $[15,16]$. In the discrete case, however, they provide more information.

Let $(X, d)$ be a compact metric space with a distance $d$. Let $f: X \rightarrow X$ be a homeomorphism of $X$ onto itself.

Recall that $f$ is expansive if there exists a constant $e>0$ such that

$$
d\left(f^{n} x, f^{n} y\right) \leq e \quad \text { for all } n \in \mathbb{Z} \text { implies } x=y
$$

and that a sequence $\left\{x_{n}\right\}_{n \in \mathbb{Z}}$ is a $\delta$-pseudo orbit if

$$
d\left(f x_{n}, x_{n+1}\right) \leq \delta \text { for all } n \in \mathbb{Z} .
$$

We say that a homeomorphism $f$ has the shadowing property or the pseudo orbits tracing property, abbreviated POTP, if and only if for every $\varepsilon>0$ there exists $\delta>0$ such that any $\delta$-pseudo orbit is $\varepsilon$-traced by some point $x \in X$, that is

$$
d\left(f^{n} x, x_{n}\right) \leq \varepsilon \quad \text { for all } n \in \mathbb{Z} .
$$

DEFINITION 1. A homeomorphism $f$ is in the class $\mathscr{H}$ if and only if $f$ is expansive and has the shadowing property.

The above class was distinguished by Walters [19]. By the results of [12] it is the same as the class of homeomorphisms having canonical or hyperbolic coordinates and 
is the same as the class of homeomorphisms generating the Smale space defined in [18].

Let $x \in X$ be an arbitrary point. We define the stable and unstable 'manifolds' at $x$ :

$$
\begin{aligned}
& W^{s}(x)=\left\{y \in X: d\left(f^{n} x, f^{n} y\right) \longrightarrow 0, \quad n \longrightarrow \infty\right\}, \\
& W^{u}(x)=\left\{y \in X: d\left(f^{n} x, f^{n} y\right) \longrightarrow 0, \quad n \longrightarrow-\infty\right\},
\end{aligned}
$$

and the local stable and unstable 'manifolds' of size $\varepsilon$ at $x$ :

$$
\begin{array}{ll}
W_{\varepsilon}^{s}(x)=\left\{y \in X: d\left(f^{n} x, f^{n} y\right) \leq \varepsilon,\right. & n \geq 0\}, \\
W_{\varepsilon}^{u}(x)=\left\{y \in X: d\left(f^{n} x, f^{n} y\right) \leq \varepsilon,\right. & n \leq 0\} .
\end{array}
$$

\section{Hyperbolic homeomorphisms}

The following definition comes from Mañé's book [8].

DEFINITION 2. A homeomorphism $f$ is hyperbolic if there exist constants $\varepsilon_{0}>0$, $K>0,0<\lambda<1$ such that

$$
\begin{array}{r}
d\left(f^{n} x, f^{n} y\right) \leq K \lambda^{n} \text { for all } x \in X, y \in W_{\varepsilon_{0}}^{s}(x) \text { and } n \geq 0 ; \\
d\left(f^{-n} x, f^{-n} y\right) \leq K \lambda^{n} \text { for all } x \in X, y \in W_{\varepsilon_{0}}^{u}(x) \text { and } n \geq 0 ;
\end{array}
$$

and there exists $\delta_{0}>0$ such that

$$
\# W_{\varepsilon_{0}}^{s}(x) \cap W_{\varepsilon_{0}}^{u}(y)=1,
$$

for all $x, y \in X$ with $d(x, y) \leq \delta_{0}$.

THEOREM 3. A homeomorphism $f$ is hyperbolic if and only if $f \in \mathscr{H}$.

PROOF. If a homeomorphism $f$ is in the class $\mathscr{H}$, then by a theorem in [12] it has hyperbolic coordinates, that is the following condition holds:

There exists $\varepsilon_{0}>0, K>0,0<\lambda<1$ such that

$$
\begin{aligned}
d\left(f^{n} x, f^{n} y\right) & \leq K \lambda^{n} d(x, y) \text { for all } x \in X, \quad y \in W_{\varepsilon_{0}}^{s}(x) \text { and } n \geq 0 ; \\
d\left(f^{-n} x, f^{-n} y\right) & \leq K \lambda^{n} d(x, y) \text { for all } x \in X, \quad y \in W_{\varepsilon_{0}}^{u}(x) \text { and } n \geq 0
\end{aligned}
$$

and there exists $\delta_{0}>0$ such that

$$
\# W_{\varepsilon_{0}}^{s}(x) \cap W_{\varepsilon_{0}}^{u}(y)=1
$$


for all $x, y \in X$ with $d(x, y) \leq \delta_{0}$.

This easily implies that $f$ is hyperbolic.

Assume that a homeomorphism $f$ is hyperbolic. Let $0<\varepsilon<\varepsilon_{0}$ be fixed. Let $n_{0}$ be a number such that $K \lambda^{n_{0}}<\varepsilon$. By continuity, there is a $\delta>0$ such that $d(x, y) \leq \delta$ and $|n| \leq n_{0}$ imply $d\left(f^{n} x, f^{n} y\right) \leq \varepsilon$. Now we have:

$$
\# W_{\varepsilon}^{s}(x) \cap W_{\varepsilon}^{u}(y)=1
$$

for all $x, y \in X$ with $d(x, y) \leq \delta_{0}$. This means that $f$ has canonical (or expansive) coordinates, so by a result in [12] it is in the class $\mathscr{H}$.

\section{Anosov diffeomorphisms}

We compare the class $\mathscr{H}$ with the class of the Anosov diffeomorphisms, which is one of the basic classes in the theory of smooth dynamical systems. So, in this section we assume that $X$ is a smooth manifold without boundary and, as before, that $X$ is compact. For any $g \in \operatorname{Diff}(X)$ we define a finite number

$$
e(g)=\sup \left\{e \geq 0: d\left(g^{n} x, g^{n} y\right) \leq e \quad \text { for all } n \in \mathbb{Z} \text { implies } x=y\right\}
$$

and notice that $e(g)>0$ if and only if $g$ is expansive.

We will say that $f \in \operatorname{Diff}(X)$ is strongly expansive, denoted by $f \in \mathscr{S} E$, if the map

$$
e: \operatorname{Diff}(X) \rightarrow[0, \infty) \text { given by } g \rightarrow e(g)
$$

is lower semi continuous in the $C^{1}$ topology at the point $f$, that is every $0<\varepsilon<e(f)$ is an expansive constant for all diffeomorphisms $g$ from a $C^{1}$ neighborhood $U$ of $f$.

The main result of this section is then

THEOREM 4. Let $f \in \operatorname{Diff}(X)$. Then $f$ is Anosov if and only if $f \in \mathscr{H} \cap \mathscr{S} E$.

This theorem will follow from more general result below. We do not repeat all definitions, referring the reader instead to standard books or monographs such as [8] or [11].

THEOREM 5. Let $f \in \operatorname{Diff}(X)$. The following conditions are equivalent.

(i) $f$ is an Anosov diffeomorphism.

(ii) $f$ is structurally stable and is expansive.

(iii) $f$ is $C^{1}$ topologically stable and strongly expansive.

(iv) $f$ is topologically stable and strongly expansive.

(v) $f$ has the shadowing property and is strongly expansive. 
We take advantage of results of Mañé and the following two propositions.

PROPOSITION 6. If $f$ is structurally stable and expansive, then it is strongly expansive.

PROOF. In view of Mañés proof of the Stability Conjecture [9], it is known that structural stability and strong structural stability are equivalent to each other. We fix $0<\varepsilon<e(f)$. Strong structural stability implies that there exists a $C^{1}$ neighborhood $U$ of $f$ such that for every $g \in U$ there is a homeomorphism $h: X \longrightarrow X$ such that

$$
d\left(h, i d_{X}\right) \leq \frac{e(f)-\varepsilon}{2}
$$

and

$$
f \circ h=h \circ g .
$$

To show that $\varepsilon$ is an expansive constant for a diffeomorphisms $g$ from $U$ assume that

$$
d\left(g^{n} x, g^{n} y\right) \leq \varepsilon \quad \text { for all } n \in \mathbb{Z} .
$$

Then we have:

$$
\begin{aligned}
d\left(f^{n} h(x), f^{n} h(y)\right) & =d\left(h\left(g^{n} x\right), h\left(g^{n} y\right)\right) \\
& \leq d\left(h\left(g^{n} x\right), g^{n} x\right)+d\left(g^{n} x, g^{n} y\right)+d\left(g^{n} y, h\left(g^{n} y\right)\right) \leq e(f),
\end{aligned}
$$

which, by expansiveness of $f$, means, $h(x)=h(y)$, and so $x=y$.

PROPOSITION 7. If $f$ is $C^{1}$ topologically stable and is a strongly expansive diffeomorphism, then it is strongly structurally stable.

PROOF. Let $0<\varepsilon<\frac{1}{2} e(f)$. $C^{1}$ topological stability provides a $C^{1}$ neighborhood $U$ of $f$ such that for any $g \in U$ there is a continuous map $h: X \longrightarrow X$ such that

$$
d\left(h, i d_{X}\right) \leq \varepsilon
$$

and

$$
f \circ h=h \circ g \text {. }
$$

We show that $h$ is a homeomorphism. If $\varepsilon$ is small enough, condition (1) guarantees that $f$ is onto $X$, see $[10$, p. 31]. We show that it is also injective. Let $h(x)=h(y)$. For all $n \in \mathbb{Z}$ we have:

$$
\begin{aligned}
d\left(g^{n} x, g^{n} y\right) & \leq d\left(g^{n} x, h\left(g^{n} x\right)\right)+d\left(h\left(g^{n} x\right), h\left(g^{n} y\right)\right)+d\left(h\left(g^{n} y\right), g^{n} y\right) \\
& \leq 2 d\left(h, i d_{X}\right)+d\left(f^{n} h(x), f^{n} h(y)\right) \leq 2 \varepsilon .
\end{aligned}
$$

One can find $U$ such that $2 \varepsilon$ is an expansive constant for all $g \in U$. Hence $x=y$. 
PROOF OF THEOREM 5. (i) is equivalent to (ii). This is a result of Mañé $[6,7]$.

(i) implies (v). By the Shadowing Lemma any Anosov diffeomorphism has the shadowing property and by Proposition 6 and condition (ii) it is strongly expansive.

(v) implies (iv). Shadowing and expansiveness imply topological stability, see [19].

(iv) implies (iii). This is obvious.

(iii) implies (ii). This follows from Proposition 7.

REMARK 8. Strong expansiveness is an essential condition. Namely, the class $\mathscr{H}$ is, generally, larger than the class of Anosov diffeomorphisms. In fact, any diffeomorphism $f$ which is topologically conjugate to some Anosov diffeomorphism $g$ but which is not Anosov itself, is a good example since shadowing and expansiveness are topological conjugacy invariants.

\section{Isolated invariant sets}

Assume once again that $X$ is a general compact metric space and let $f \in \mathscr{H}$.

We will denote by $c(\delta)$ a positive number such that

$$
d(x, y) \leq c(\delta) \text { implies } d(f x, f y) \leq \delta, d\left(f^{-1} x, f^{-1} y\right) \leq \delta .
$$

Let $K \subset X$ be a nonempty closed invariant set. $K$ is isolated or locally maximal if there is a neighborhood $U$ of $K$ such any invariant subset of $U$ must be contained in $K$.

PROPOSITION 9. Let $K$ be a closed invariant set. The following conditions are equivalent.

(i) The restriction $f \mid K \in \mathscr{H}$.

(ii) There is a number $e_{K}>0$ such that:

$$
d\left(f^{n} x, K\right) \leq e_{K} \quad \text { for all } n \in \mathbb{Z} \text { implies } x \in K .
$$

(iii) $K$ is an isolated set.

ProOF. (i) implies (ii). Let $e>0$ be an expansive constant of $f$. By condition (i) we can find $\delta>0, \delta<e$ such that any $\delta$-p.o. in $K$ is $e / 2$ traced by a point from $K$. We set $e_{K}=\min (c(\delta / 2), \delta / 2)$. Assume that for some $x \in X$ we have $d\left(f^{n} x, K\right) \leq e_{K}$, for all $n \in \mathbb{Z}$. There are points $x_{n} \in K$ such that $d\left(f^{n} x, x_{n}\right) \leq e_{K}$. These points form a $\delta$-p.o. because

$$
d\left(f x_{n}, x_{n+1}\right) \leq d\left(f x_{n}, f f^{n} x\right)+d\left(f^{n+1} x, x_{n+1}\right) \leq \delta .
$$


This $\delta$-p.o. is $e / 2$ traced by a point $y$ from $K$. By the triangle inequality:

$$
d\left(f^{n} x, f^{n} y\right) \leq d\left(f^{n} x, x_{n}\right)+d\left(x_{n}, f^{n} y\right) \leq e_{K}+\frac{e}{2} \leq e .
$$

By expansiveness, $x=y \in K$.

(ii) implies (iii). It is clear that $U=\left\{x \in X: d(x, K) \leq e_{K}\right\}$ is such a neighborhood of $K$.

(iii) implies (i). Clearly $f \mid K$ is expansive. We show it has the shadowing property. Let $\varepsilon>0$ be such that $d(x, K)<\varepsilon$ implies $x \in U$. By the shadowing of $f$ (on the whole space $X$ ) we choose $\delta$ corresponding to this $\varepsilon$. Let $\left\{x_{n}\right\}_{n \in \mathbb{Z}}$ be a $\delta$-p.o. contained in $K$. We get a point $x \in X$ such that $d\left(f^{n} x, x_{n}\right) \leq \varepsilon$, for all $n \in \mathbb{Z}$. Now $L=K \cup\left\{f^{n} x\right\}_{n \in \mathbb{Z}}$ is clearly invariant and contained in $U$. Hence, by (iii), $L \subset K$ and therefore $x \in K$, which completes the proof.

Let $K \subset X$ be an invariant set. We define the stable and unstable 'manifolds' at $K$ :

$$
\begin{aligned}
& W^{s}(K)=\left\{x \in X: d\left(f^{n} x, K\right) \longrightarrow 0, n \longrightarrow \infty\right\}, \\
& W^{u}(K)=\left\{x \in X: d\left(f^{n} x, K\right) \longrightarrow 0, n \longrightarrow-\infty\right\},
\end{aligned}
$$

and the local stable and unstable 'manifolds' of size $\varepsilon$ at $K$ :

$$
\begin{aligned}
& W_{\varepsilon}^{s}(K)=\left\{x \in X: d\left(f^{n} x, K\right) \leq \varepsilon, n \geq 0\right\}, \\
& W_{\varepsilon}^{u}(K)=\left\{x \in X: d\left(f^{n} x, K\right) \leq \varepsilon, n \leq 0\right\} .
\end{aligned}
$$

PROPOSITION 10. Let $K \subset X$ be a closed invariant isolated set. Then:

(i) For every $\varepsilon>0$ there exists $\delta>0$ such that

$$
W_{\delta}^{s}(K) \subset \bigcup_{x \in K} W_{\varepsilon}^{s}(x) .
$$

(ii) For any small $\delta>0$

$$
W_{\delta}^{s}(K) \subset W^{s}(K)
$$

(iii)

$$
W^{s}(K)=\bigcup_{x \in K} W^{s}(x)
$$

PROOF. (i). For $\varepsilon>0$ pick $\delta_{1}>0$ such that any $\delta_{1}$-p.o. in $K$ is $\varepsilon$-traced by some point in $K$, and then pick $\delta=c\left(\delta_{1} / 2\right), \delta \leq \min \left(\delta_{1} / 2, \varepsilon / 2\right)$. Let $x \in W_{\delta}^{s}(K)$. Then $d\left(f^{n} x, x_{n}\right) \leq \delta$ for $n \geq 0$, where $x_{n} \in K$. The sequence

$$
\ldots, f^{-2} x_{0}, f^{-1} x_{0}, x_{0}, x_{1}, x_{2}, x_{3}, \ldots
$$


is a $\delta_{1}$-p.o., as $d\left(f x_{0}, x_{1}\right) \leq d\left(f x_{0}, f x\right)+d\left(f x, x_{1}\right)$. It is $\varepsilon / 2$-traced by some point $y \in K$ and hence, by the triangle inequality, $x \in W_{\varepsilon}^{s}(y)$.

(ii). It is known (and using compactness arguments is not hard to prove) that for any $x \in X, W_{\varepsilon}^{s}(x) \subset W^{s}(x)$, where $\varepsilon<e$ and $e$ is an expansive constant of $f$. Now it is enough to choose $\delta$ corresponding to such an $\varepsilon$ in terms of (i) that we have just proved.

(iii). Let $x \in W^{s}(K)$. Let $\varepsilon \leq e$ and choose $\delta$ corresponding to this $\varepsilon$ in terms in (i). By the definition of $W^{s}(K)$ there is $n$ such that $f^{n} x \in W_{\delta}^{s}(K)$. By (i) there is a point $y \in K$ such that $f^{n} x \in W_{\delta}^{s}(y) \subset W^{s}(y)$. Hence, $x \in W^{s}\left(f^{-n} y\right)$. Since $K$ is invariant, $f^{-n} y \in K$.

REMARK 11. We will also use the dual statement to Proposition 10 which can be expressed in terms of the unstable 'manifolds'.

THEOREM 12. Let $K \subset X$ be a closed invariant isolated set. Let $\varepsilon>0$ be a small number. Then the following conditions are equivalent.

(i) $K \subset$ int $W_{\varepsilon}^{s}(K)$.

(ii) There is a neighborhood $G$ of $K$ such that $f G \subset G$ and $\bigcap_{n=0}^{\infty} f^{n} G=K$.

(iii) $W^{u}(K)=K$.

(iv) $W_{\varepsilon}^{u}(K)=K$.

ProOF. Let $\varepsilon>0$ be small such that $W_{\varepsilon}^{s}(K) \subset W^{s}(K)$ and $\varepsilon \leq e_{K}$, where $e_{K}$ was defined in Proposition 9.

(i) implies (ii). Let $G_{1}$ be an open set such that $K \subset G_{1} \subset W_{\varepsilon}^{s}(K)$. Define $G=\bigcup_{n=0}^{\infty} f^{n} G_{1}$. It is clear that $G$ is an open neighborhood of $K$ and $f G \subset G$. Also, for $x \in G$ we have $d(x, K)<\varepsilon$. Let $x \in \bigcap_{n=0}^{\infty} f^{n} G$. It implies $f^{n} x \in G$ for all $n \leq 0$. In particular, $x \in G$ and by the inclusion $f G \subset G$, it follows that $f^{n} x \in G$, for all $n \in \mathbb{Z}$. So $d\left(f^{n} x, K\right) \leq \varepsilon \leq e_{K}$ for all such $n$ s. Thus $x \in K$.

(ii) implies (iii). Let $x \in W^{u}(K)$. By the definition of $W^{u}(K)$, there is an integer $m$ such that $f^{n} x \in G$ for all $n \leq m$ or, equivalently, $f^{m} x \in \bigcap_{n=0}^{\infty} f^{n} G=K$. Since $K$ is invariant, $x \in K$.

(iii) implies (iv). This follows from the dual version of Proposition 10(ii).

(iv) implies (i). Let $\delta>0$ corresponds to $\min (\varepsilon, c(\varepsilon))$ in terms of the shadowing property. Let $x \in K$ and $y \in B(x, \delta)$ be fixed. The sequence $\ldots, f^{-2} x, f^{-1} x, y, f y$, $f^{2} y, \ldots$ is a $\delta$-p.o. and is $c(\varepsilon)$-traced by some point $z$. Then we have, $d\left(f^{n} x, f^{n} z\right) \leq$ $\varepsilon$ for all $n \leq 0$, and so $z \in W^{u}(x) \subset W^{u}(K)=K$. Also, $d\left(f^{n} z, f^{n} y\right) \leq \varepsilon$ for all $n \geq 0$ and so $y \in W_{\varepsilon}^{s}(z)$. As $z \in K$, we see that $y \in W_{\varepsilon}^{s}(K)$. We have proved that $B(x, \delta) \subset W_{\varepsilon}^{s}(K)$ for every $x \in K$, which completes the proof. 
REMARK 13. One can state the dual theorem to the above theorem by interchanging the role of the stable and unstable manifolds. Moreover, both Theorem 12 and its dual counterpart describe the disjoint situations in the case the set $K$ is non-isolated in the space $X$, that is any neighborhood of $K$ contains points from $X \backslash K$. In fact, conditions (ii) and the condition dual to the condition (iv), namely $W_{\varepsilon}^{s}(K)=K$, exclude each other.

Having in mind basic sets or periodic orbits we will assume that the set $K$ satisfies the following condition:

AsSUMPTION. The set $K \subset X$ is closed isolated invariant and

For every $\varepsilon>0$ and $x, y \in K$

(B)

$$
\begin{aligned}
& \text { there exists an } \varepsilon \text {-chain }\left(x=x_{0}, \ldots, x_{n}=y\right) \text { in } K \text {, that is } \\
& d\left(f x_{i}, x_{i+1}\right) \leq \varepsilon \text {, for } i=0, \ldots, n-1 \text {, and all } x_{i} \in K .
\end{aligned}
$$

In the next Section we will show that any basic set satisfies the above condition (B). Notice that any periodic orbit satisfies this condition. In fact, the only nontrivial thing to check is that a periodic orbit is isolated, but this follows from the observation that any homeomorphism on its periodic orbit has the shadowing property and one can apply Proposition 9.

Under the above Assumption we may extend Theorem 12 as follows:

THEOREM 14. Let $K \subset X$ satisfy the assumption. Let $\varepsilon>0$ be a small number. Then, the following conditions are equivalent.

(i) $K \subset$ int $W_{\varepsilon}^{s}(K)$.

(ii) There is a neighborhood $G$ of $K$ such that $f G \subset G$ and $\bigcap_{n=0}^{\infty} f^{n} G=K$.

(iii) $W^{u}(K)=K$.

(iv) $W_{\varepsilon}^{u}(K)=K$.

(v) int $W^{s}(K) \neq \emptyset$.

(vi) $K \subset$ int $W^{s}(K)$

(vii) $W^{s}(K)$ is open.

(viii) int $W_{\varepsilon}^{s}(K) \neq \emptyset$.

PROOF. Since the first four conditions are equivalent to each other by Theorem 12, it is enough to prove that the following implications are true:

(v) implies (vi) implies (vii) implies (i) implies (viii) implies (v).

(v) implies (vi). Let $a$ and $\varepsilon_{1}>0$ be such that $B\left(a, \varepsilon_{1}\right) \subset W^{s}(K)$. Let $\varepsilon>0$ be such that $W_{\varepsilon}^{s}(K) \subset W^{s}(K)$, using Proposition 10(ii). Let $\delta \leq \min \left(\varepsilon_{1}, \varepsilon / 2\right)$ 
be chosen such that any $\delta$-p.o. in $X$ is $\min \left(\varepsilon_{1}, \varepsilon / 2\right)$-traced. It is enough to show that $B(K, c(\delta)) \subset W^{s}(K)$. Let then $y \in B(K, c(\delta))$, so $d(y, x) \leq c(\delta)$ with some $x \in K$. Since $a \in W^{s}(K)$ we have $b \in K$ and $n>0$ such that $d\left(f^{n} a, b\right) \leq \delta$. Let $\left(b=y_{0}, \ldots, y_{k}=x\right)$ be a $\delta$-chain provided by the assumption. Define a $\delta$-p.o.

$$
x_{i}= \begin{cases}f^{i} a & \text { for } i \leq n-1, \\ y_{i-n} & \text { for } n \leq i \leq n+k, \\ f^{i-(n+k)} y & \text { for } n+k<i .\end{cases}
$$

This is $\min \left(\varepsilon_{1}, \varepsilon / 2\right)$-traced by some point $z$. In particular,

$$
z \in B\left(a, \varepsilon_{1}\right) \subset W^{s}(K),
$$

hence for large $i$, say $i \geq i_{0}, d\left(f^{i} z, K\right) \leq \varepsilon / 2$. On the other hand, for $i>n+k$, $d\left(f^{i} z, f^{i-(n+k)} y\right) \leq \varepsilon / 2$, and by the triangle inequality, $d\left(f^{j} y, K\right) \leq \varepsilon$ for $j \geq j_{0}=$ $n+k+i_{0}$. This means that $f^{j_{0}} y \in W_{\varepsilon}^{s}(K) \subset W^{s}(K)$. Hence, $y \in W^{s}(K)$.

(vi) implies (vii). If $x \in W^{s}(K)$, then by (vi) there is $N$ and an open set $U$ such that $f^{N} x \in U \subset W^{s}(K)$ such. Continuity of $f^{N}$ completes the proof.

(vii) implies (i). We argue by contradiction. To do so we assume that any neighborhood of $K$ contains points which do not belong to $W_{\varepsilon}^{s}(K)$. On the other hand, since $K$ is compact, there is $\varepsilon_{1}>0$ such that $K \subset B\left(K, \varepsilon_{1}\right) \subset W^{s}(K)$. Let $\delta>0$ correspond to $\min \left(\varepsilon_{1}, \varepsilon / 2\right)$ by the shadowing property. By our assumption we find points $a, y$ such that $a \in K, d(a, y) \leq \min \left(\varepsilon_{1}, c(\delta)\right)$, and $d\left(f^{N} y, K\right) \geq \varepsilon$ for some positive $N$. By the choice of $\varepsilon_{1}, y \in W^{s}(K)$. So, we have a number $k>N$ such that $d\left(f^{k} y, b\right) \leq \delta$, with some $b \in K$. We define a $\delta$-p.o. to be a periodic sequence built of blocks of the form:

$$
a, f y, \ldots, f^{k-1} y, b, y_{1}, \ldots, y_{m-1},
$$

where $\left(b=y_{0}, \ldots, y_{m}=a\right)$ is a $\delta$-chain provided by the assumption. This $\delta$-p.o. is $\min \left(\varepsilon_{1}, \varepsilon / 2\right)$ traced by some point $x$. Now, $x \in B\left(K, \varepsilon_{1}\right) \subset W^{s}(K)$, which means that $d\left(f^{n} x, K\right) \longrightarrow 0$ as $n \longrightarrow \infty$. On the other hand the orbit of $x$ visits the ball $B\left(f^{N} y, \varepsilon / 2\right)$ infinitely many times which, by the triangle inequality, means that it leaves the neighborhood $B(K, \varepsilon / 2)$ infinitely many times, which is a contradiction.

(i) implies (viii) is trivial.

(viii) implies (v). It follows from Proposition 10(ii).

REMARK 15. The theorem we have just proved has a dual version when we change the role of the stable and unstable manifolds.

REMARK 16. Since certain conditions in the above theorem do not depend on $\varepsilon$, then neither do the others. In other words, if, for example, condition (i) holds for some small $\varepsilon$, then it holds for every small $\varepsilon$. 
REMARK 17. By the above Remark, Theorem 14 can be expressed in terms of the classical theory of stability of sets in the theory of dynamical systems, see [3]. In fact, condition (i) means stability of $K$, condition (ii) that $K$ is an attractor in the sense of Conley, condition (iii) or (iv) that $K$ is a repellor, condition (v) that $K$ attracts from some open set, condition (vi) that $K$ is an attractor, condition (vii) that $K$ has an open set of attractivity. However, condition (viii), does not have such a natural interpretation. Theorem 14 says that all these notions mentioned above are equivalent to each other under the Assumption.

Let the set $K$ satisfy the Assumption and be non-isolated in the space $X$, see Remark 13. According to that remark, we can distinguished three types of behavior near the set $K$ : first, if any of the conditions stated in Theorem 12 or Theorem 14 (and then all remaining conditions) is satisfied; second, if any of the conditions from the dual versions to those theorems (and then all remaining conditions) is satisfied; and third, if all the above conditions fail. We will say that the set $K$ is respectively a sink, a source or a saddle.

Also, in a similar way, any point nonisolated in $X$ can be treated as a sink, a source or a saddle, see [13, 14, 17]. In fact, in [13] a counterpart of Theorem 14 is proved and it has its dual version when we change the role of the stable and unstable manifolds and consider negative limit set instead of positive limit set. We recall this result here.

THEOREM 18. [13] Let $\varepsilon>0$ be a small number, $x \in K$ and $y$ a positive limit point of $x(y \in \omega(x))$. Then, the following conditions are equivalent.

(i) int $W^{s}(x) \neq \emptyset$.

(ii) $x \in$ int $W^{s}(x)$.

(iii) $W^{s}(x)$ is open.

(iv) $x \in \operatorname{int} W_{\varepsilon}^{s}(x)$.

(v) int $W_{\varepsilon}^{s}(x) \neq \emptyset$.

(vi) $W_{\varepsilon}^{u}(x) \cap H=\{x\}$, where $H$ is a neighborhood of $x$.

(vii) $W_{\varepsilon}^{u}(y)=\{y\}$

(viii) $W^{u}(y)=\{y\}$.

(ix) $\omega(x)=\left\{y, \ldots, f^{k-1} y\right\}$ is a periodic orbit, and there is a neighborhood $U$ of $x$ such that $f U \subset U$ and $\bigcap_{n=0}^{\infty} f^{k n} U=\{y\}$.

As we have mentioned before, any periodic orbit satisfies the Assumption so it may be treated as a sink, a source or a saddle. The following proposition answers the natural question.

PROPOSITION 19. Let $x \in X$ be a periodic point, nonisolated in the space $X$ and let $K=\left\{x, \ldots, f^{k-1} x\right\}$ be its periodic orbit. Then $x$ is a sink, a source or a saddle, if and only if $K$ is a sink, a source or a saddle, respectively. 
PROOF. Let us notice that by the Proposition 10(iii) we have

$$
W^{s}(K)=W^{s}(x) \cup \cdots \cup W^{s}\left(f^{k-1} x\right)
$$

and it is clear that all $W^{s}\left(f^{i} x\right)$ are homeomorphic and pairwise disjoint. Hence, $W^{s}(K)$ is open if and only if $W^{s}(x)$ is open, which proves the proposition for the case of sink. The case of source is similar and the case of saddle follows the previous cases.

The above proposition and Theorem 18 imply the following corollary.

COROLLARY 20. Let $x \in X$ be a nonisolated point in the space $X$. Then $x$ is a sink if and only if $\omega(x)$ is a periodic orbit and is a sink. A similar statement holds for a source.

\section{Basic sets}

Denote by $\operatorname{Per}(f)$ the set of all periodic points of $f$ and by $\Omega(f)$ the set of all nonwandering points of $f$. It is known that the above sets are nonempty invariant, and $\Omega(f)$ is closed with $\operatorname{Per}(f)$ is dense in $\Omega(f)$. Also $\Omega(f)=C R(f)$, where $C R(f)$ denotes the set of all chain recurrent points, see [13]. The restriction $f \mid \Omega(f) \in \mathscr{H}$ (see [1, 14]). Moreover, repeating Bowen's proof for Axiom A diffeomorphisms, one has the following Spectral Decomposition Theorem (see $[2,14])$.

THEOREM 21.

$$
\Omega(f)=\Omega_{1} \cup \cdots \cup \Omega_{k},
$$

where the $\Omega_{1}, \ldots, \Omega_{k}$ are said to be basic sets. They are closed invariant and pairwise disjoint and the restriction $f \mid \Omega_{i}$ is topologically transitive, for $i=1, \ldots, k$.

Moreover, any basic set has a decomposition:

$$
\Omega_{i}=\Omega_{i, 1}, \ldots, \Omega_{i, k_{i}},
$$

where the $\Omega_{i, j}$ are closed pairwise disjoint and $f \Omega_{i, 1}=\Omega_{i, 2}, \ldots, f \Omega_{i, k_{i}}=\Omega_{i, 1}$. The restrictions $f^{k_{i}} \mid \Omega_{i, j}$ are topologically mixing on each $\Omega_{i, j}, i=1, \ldots, k, j=$ $1, \ldots, k_{i}$.

We show that any basic set satisfies the assumption from Section 4 and therefore in the case it is non-isolated in the space $X$ can be treated as a sink or a source or a saddle. In fact, we have already mentioned the restriction $f \mid \Omega(f) \in \mathscr{H}$. In particular, the restriction of $f$ to any basic set has the shadowing property, so by Proposition 9 any basic set is an isolated invariant set. We have to show: 
Proposition 22. Let $K$ be a basic set. Then $K$ satisfies condition (B) of the assumption.

PRoOF. Let $x, y \in K$ and $\varepsilon>0$ be fixed. We have to find an $\varepsilon$-chain from $x$ through $y$. Since $K \subset C R(f)$, the case $x=y$ is obvious. So we assume that $x \neq y$. Let $\delta=c(\varepsilon) / 2, \delta<\varepsilon$. We have a dense orbit in $K$, so there is a point $w \in K$ and an integer $M \neq 0$ such that $d(x, w) \leq \delta$ and $d\left(y, f^{M} w\right) \leq \delta$. If $M>0$ then the sequence $\left(x, f w, \ldots, f^{M-1} w, y\right)$ is an $\varepsilon$-chain as required.

Assume $M<0$. Let $z=f^{M} w$ and $m=-M$. Now we have

$$
d(y, z) \leq \varepsilon / 2 \text { and } d\left(x, f^{m} z\right) \leq c(\varepsilon) / 2 .
$$

Since periodic points are dense in $K$ we can find a periodic point $p \in K$ such that

$$
d(z, p) \leq \varepsilon / 2 \text { and } d\left(f^{m} z, f^{m} p\right) \leq c(\varepsilon) / 2 .
$$

There is $k>1$ such that $f^{m+k} p=p$. By the above estimations we have:

$$
d\left(x, f^{m} p\right) \leq d\left(x, f^{m} z\right)+d\left(f^{m} z, f^{m} p\right) \leq c(\varepsilon),
$$

and

$$
d(p, y) \leq d(p, z)+d(z, y) \leq \varepsilon .
$$

So, the sequence $\left(x, f^{m+1} p, \ldots, f^{m+k-1} p, y\right)$ is such a required $\varepsilon$-chain.

The following is, to some extent, a converse statement to the above proposition:

PROPOSITION 23. If the set $K$ satisfies the Assumption and is a sink or a source, then $K$ is a basic set.

PROOF. Condition (B) implies that any point in $K$ is chain recurrent, so $K \subset$ $C R(f)=\Omega(f)$. Moreover, the same condition yields that $K$ cannot intersect two distinct basic sets, so there is such a basic set $\Omega_{i}$ that $K \subset \Omega_{i}$.

We want to show the opposite inclusion but first we claim that for any two points $x, y$ from $\Omega_{i}$ and any $\varepsilon>0$ there is a periodic orbit $\left(p, \ldots, f^{k-1} p\right)$ such that $d(x, p) \leq \varepsilon$ and $d\left(y, f^{i} p\right) \leq \varepsilon$, with some $0 \leq i \leq k-1$. We can assume that $\varepsilon<e / 2$, where $e>0$ is an expansive constant. Choose $\delta$ corresponding to this $\varepsilon$ in the shadowing property and consider two $\delta$-chains provided by Proposition 22:

$$
x=x_{0}, \ldots, x_{k}=y \text { and } y=y_{0}, \ldots, y_{l}=x .
$$

Define a periodic $\delta$-p.o.; 
It is $\varepsilon / 2$-traced by some point $p$ and, also, by $f^{k+l-1} p$. By expansiveness, $f^{k+l-1} p=$ $p$, which proves the claim.

Assume that there is a point $x \in \Omega_{i} \backslash K$. As $K$ is closed, there is $\varepsilon>0$ such that $B(x, \varepsilon) \cap K=\emptyset$. Pick a point $y \in K \cap \Omega_{i}$. As $K$ is a sink, $W^{s}(K)$ is open and we may decrease $\varepsilon$ so that $B(y, \varepsilon) \subset W^{s}(K)$. From the assertion we have a periodic orbit such that some element in it lies in $B(x, \varepsilon)$ and hence is not in $K$. On the other hand this orbit meets the stable 'manifold' $W^{s}(K)$, hence is contained in $K$, which is a contradiction.

In the sequel we will assume that the nonwandering set $\Omega(f)$ is a proper subset of $X$ and the all basic sets are non-isolated in the space $X$. This last assumption is a quite natural one. In fact, the dynamics on an isolated basic set is independent of the dynamics on the remaining part of the space.

THEOREM 24. Let $\Omega(f) \neq X$. Assume that any basic set is non-isolated in the space $X$. Then:

(i) $f$ has the no-cycle property. In particular, there are at least two basic sets.

(ii) int $\Omega(f)=\emptyset$.

(iii) Among basic sets there is at least one sink. The set of points whose positive orbits tend to some basic set which is a sink is dense and open in the space $X$. The corresponding statement is also true for the source case.

(iv) If the space $X$ is connected and locally connected, then any basic set which is a sink or a source is uncountable.

PROOF. (i). It is the same as the proof of the corresponding result for Axiom A diffeomorphisms, [5].

(ii). If int $\Omega_{i} \neq \emptyset$, for some basic set $\Omega_{i}$, then both $W^{s}\left(\Omega_{i}\right)$ and $W^{u}\left(\Omega_{i}\right)$ have nonempty interiors, hence $\Omega_{i}$ is a sink and a source at the same time, which is absurd.

(iii) We will show that $X=W^{s}\left(\Omega_{1}\right) \cup \ldots \cup W^{s}\left(\Omega_{k}\right)$, where $\Omega_{1}, \ldots, \Omega_{k}$ are all basic sets. Having proved this we argue as follows. Fix any open set $U \subset X$. Since for any $\Omega_{i}, W^{s}\left(\Omega_{i}\right)=\bigcup_{n=0}^{\infty} f^{-n} W_{\varepsilon}^{s}\left(\Omega_{i}\right)$, the set $U$ is contained in the countable union of the closed sets of the form $f^{-n} W_{\varepsilon}^{s}\left(\Omega_{i}\right)$. By the Baire Category Theorem and the continuity of $f^{n}$, there is a basic set for which int $W^{s}\left(\Omega_{i}\right) \neq \emptyset$, so this basic set is a sink and its stable 'manifold' contains a point from $U$. The union of the stable 'manifolds' of sinks is clearly open.

To prove our first claim notice that the positive limit set of every point is contained in $\Omega(f)$. The proof will be complete if we show that the positive limit set is contained in a single basic set. We argue by contradiction. So, let $x, y, z \in X$ be such that $y \in \omega(x) \cap \Omega_{1}, z \in \omega(x) \cap \Omega_{2}$, where $\Omega_{1}, \Omega_{2}$ are distinct basic sets. Fix $\varepsilon>0$ so small that $2 \varepsilon<e, \varepsilon<\min \left\{\operatorname{dist}\left(\Omega_{i}, \Omega_{j}\right): \Omega_{i}, \Omega_{j}\right.$ basic sets $\}$, where $e$ is an expansivity 
constant. Let $\delta$ correspond to $\varepsilon$ in terms of the shadowing property. There are positive numbers $n_{1}<n_{2}<n_{3}$ such that;

$$
d\left(f^{n_{1}} x, y\right)<c(\delta), d\left(f^{n_{2}} x, z\right)<c(\delta), d\left(f^{n_{3}} x, y\right)<c(\delta) .
$$

We form a periodic $\delta$-p.o. as follows

$$
\ldots, y, f^{n_{1}+1} x, \ldots, f^{n_{2}-1} x, z, f^{n_{2}+1} x, \ldots, f^{n_{3}-1} x, y, \ldots
$$

This orbit is $\varepsilon$-traced by a point $p$, which by expansiveness is periodic, so the whole orbit of $p$ is contained in a single basic set. On the other hand we have points on this orbit in $\varepsilon$-neighborhoods of $\Omega_{1}$ and $\Omega_{2}$. By the choice of $\varepsilon$ it is absurd.

(iv). We use a result from [17] which says that under our assumption every point in $X$ is a saddle. We argue by contradiction and assume that the basic set $K$ is countable and a sink. From Proposition 10(i) we have:

$$
W_{\delta}^{s}(K) \subset \bigcup_{x \in K} W_{\varepsilon}^{s}(x)
$$

with appropriate small $\varepsilon$ and $\delta$. By the result mentioned above any set $W_{\varepsilon}^{s}(x)$ is nowhere dense, so by the Baire Category Theorem the interior of its countable union is empty. This is a contradiction to condition (viii) in Theorem 14.

\section{References}

[1] N. Aoki, 'On homeomorphisms with pseudo-orbit tracing property', Tokyo J. Math. 6 (1983), 329-334.

[2] — 'Topological dynamics', in: Topics in general topology (eds. K. Morita and J. Nagata; Elsevier Science Publishers, New York, 1989) pp. 625-739.

[3] N. Bhatia and G. Szego, Stability theory of dynamical systems (Springer, Berlin, 1970).

[4] R. Bowen, Equilibrium states and the ergodic theory of Anosov diffeomorphisms, Lectures Notes in Math. 470 (Springer, Berlin, 1975).

[5] —_, On Axiom A diffeomorphisms, Regional Conference Series in Math 35 (Amer. Math. Soc., Providence, 1978).

[6] R. Mañe, 'Quasi Anosov diffeomorphisms', in: Dynamical systems, Warwick, 1974 (ed. A. Manning), Lectures Notes in Math. 468 (Springer, Berlin, 1975) pp. 27-29.

[7] —_, 'Expansive diffeomorphisms', in: Dynamical systems, Warwick, 1974 (ed. A. Manning), Lectures Notes in Math. 468 (Springer, Berlin, 1975) pp. 162-174.

[8] - Ergodic theory and differentiable dynamics (Springer, Berlin, 1987).

[9] __. 'A proof of the $C^{1}$ stability conjecture', Inst. Hautes Études Sci. Publ. Math. 66 (1988), $161-210$.

[10] J. Munkres, Elementary differential topology (Princeton University Press, 1963).

[11] Z. Nitecki, Differentiable dynamics (M.I.T. Press, 1971). 
[12] J. Ombach, 'Equivalent conditions for hyperbolic coordinates', Topology Appl. 23 (1986), 87-90.

[13] —_, 'Consequences of the pseudo orbits tracing property and expansiveness', $J$. Australian Math. Soc. (Series A) 43 (1987), 301-313.

[14] _ _ 'Expansive homeomorphisms with the pseudo orbits tracing property', Polish Academy of Sciences, preprint 383, 1987.

[15] —_, 'Sinks, sources and saddles for expansive flows with the pseudo-orbits tracing property', Ann. Polon. Math. 53 (1991), 238-252.

[16] _ 'Saddles for expansive flows with the pseudo orbits tracing property', Ann. Polon. Math. 56 (1991), 37-48.

[17] W. Reddy and L. Robertson, 'Sources, sinks and saddles for expansive homeomorphisms with canonical coordinates', Rocky Mountain J. Math. 17 (1987), 673-681.

[18] D. Ruelle, Thermodynamic formalism (Addison-Wesley, Reading, 1978).

[19] P. Walters, 'On the pseudo orbit tracing property and its relationship to stability', in: The structure of attractors in dynamical systems (eds. J. C. Martin, N. G. Markley and W. Perrizo), Lecture Notes in Math. 668 (Springer, Berlin, 1978) pp. 231-244.

[20] K. Sakai, 'Hyperbolic metrics for expansive homeomorphisms', Topology Appl. 63 (1995), 263266.

\section{Instytut Matematyki}

Uniwersytet Jagielloński

ul. Reymonta 4, 30059 Kraków

Poland

e-mail: ombach@im.uj.edu.pl 